\title{
PROXIMITY EFFECTS AND CURIE TEMPERATURE ENHANCEMENT IN Co/EuS AND Fe/EuS MULTILAYERS
}

\author{
B. LEWITZ ${ }^{1}$, A. STRAUB ${ }^{1}$, V. KAPAKLIS ${ }^{2}$, P. POULOPOULOS ${ }^{1,3,4, *}$, \\ A. DELIMITIS ${ }^{5}$, S.D. PAPPAS ${ }^{3}$ and P. FUMAGALLI ${ }^{1}$ \\ ${ }^{1}$ Institut für Experimentalphysik, Freie Universität Berlin, Arnimallee 14, D-14195 Berlin-Dahlem, \\ Germany \\ ${ }^{2}$ Department of Physics and Astronomy, Uppsala University, Box 516, SE-751 20 Uppsala, Sweden \\ ${ }^{3}$ Laboratory of High-Tech Materials, School of Engineering, University of Patras, 26504 Patras, \\ Greece \\ ${ }^{4}$ Materials Science Department, University of Patras, 26504 Patras, Greece \\ ${ }^{5}$ Chemical Process \& Energy Resources Institute (CPERI), Centre for Research \& Technology Hellas \\ (CERTH), 57001 Thermi, Thessaloniki, Greece
}

\begin{abstract}
Two identical Co/EuS and Fe/EuS multilayers of 6 periods each and with individual layers of about $4 \mathrm{~nm}$ thick are grown by e-beam evaporation under ultrahigh vacuum conditions. The films show polycrystalline structure with a grain size limited by the individual layer thickness. Both multilayers consist of almost continuous layers with some roughness. The surface peakto-peak roughness is about 4-5 $\mathrm{nm}$. Magnetization measurements and calculations of the loops based on a Stoner-Wohlfarth-like model allow us to determine the direct antiferromagnetic exchange coupling constant between the $3 d$ metal and EuS at $5 \mathrm{~K}$. Both samples show strong enhancement of the Curie Temperature of EuS up to at least $50 \mathrm{~K}$ with a EuS magnetization tail, which persists up to about $100 \mathrm{~K}$. The $\mathrm{J}=7 / 2$ character of the EuS layers is shown to be responsible for the large Curie temperature enhancement.
\end{abstract}

Keywords: magnetic multilayers, EuS, Co, Fe, spintronics, antiferromagnetic coupling, proximity effects, Curie temperature

* Corresponding author: P. Poulopoulos, Tel. +30-2610-997884

email: poulopoulos@zedat.fu-berlin.de AND poulop@upatras.gr 


\section{Introduction}

Nanostructured magnetic materials are important nowadays in technological applications such as magnetic recording, sensors, actuators and spintronics. ${ }^{1-4}$ In the next generation of magnetic materials for nanodevices, the electron spin will be used to store information and charge currents will be replaced by spin currents. For this purpose, ferromagnetic semiconductors at room temperature are needed. The majority of the work on magnetic semiconductors was focused on diluted magnetic semiconductors. ${ }^{3,5,6}$ However, recent experiments with element specificity have demonstrated that, for example, there is no sign of intrinsic ferromagnetic interaction between Co substitution (dopant) ions in $\mathrm{ZnO}$. The $\mathrm{Co}$ ions show purely paramagnetic or superparamagnetic response. ${ }^{7,8}$ This observation leaves a lot of open field for research on natural ferromagnetic semiconductors for future application in spintronics industry. Europium sulfide $(\mathrm{EuS})$ is a natural ferromagnetic semiconductor. That is, as compared to layered systems ferromagnet/semiconductor, it presents the advantage that the spin-polarized electrons are created within the semiconductor itself. The disadvantage of EuS is the low Curie temperature $\mathrm{T}_{\mathrm{C}} \sim 16.6 \mathrm{~K}$. To be useful for applications EuS should be ferromagnetic at room temperature (R.T.). An early work by Fumagalli et al. showed that EuS nanospheres in a Co matrix are ferromagnetic up to $160 \mathrm{~K} .{ }^{9}$ Successive works on EuS/Co bilayers and trilayers with EuS layers thicker than $3.5 \mathrm{~nm}$ showed also some $\mathrm{T}_{\mathrm{C}}$ enhancement of EuS due to proximity to Co, but no convincing R.T ferromagnetism of EuS was provided. ${ }^{10-12}$

In this work, we perform a comparative study of two identical Co/EuS and Fe/EuS multilayers with respect to growth, proximity effects, exchange coupling and $\mathrm{T}_{\mathrm{C}}$ enhancement. Both samples possess slightly reduced magnetic moments of the $3 d$ metals and significantly reduced magnetic moments of $\mathrm{EuS}$ at a temperature of $5 \mathrm{~K}$. This is attributed to the problem of mixed valency of EuS layers grown at relatively low substrate temperatures $\left(<200{ }^{\circ} \mathrm{C}\right) .{ }^{13}$ 
Magnetization loops at $5 \mathrm{~K}$ show a shape, which is characteristic for antiferromagnetically coupled $3 d$ magnetic metal and EuS layers. Calculations of the magnetization loops via a Stoner-Wolfarth-like model, ${ }^{14,15}$ allow for a precise determination of the exchange coupling constants, which are larger than previous values published for $\mathrm{Co} / \mathrm{EuS}$ and $\mathrm{Fe} / \mathrm{EuS}$ bilayers. ${ }^{16-}$

${ }^{18}$ Both multilayers show the same strong enhancement of EuS $\mathrm{T}_{\mathrm{C}}$ up to $50 \mathrm{~K}$ with a EuS magnetization tail persisting up to about $100 \mathrm{~K}$. Calculations of the magnetic response above $\mathrm{T}_{\mathrm{C}}$ are performed following a Brillouin function for a $\mathrm{J}=7 / 2$ system, like the $\mathrm{EuS}$ and a common $\mathrm{J}=1 / 2$ magnet, like e.g. Ni. The calculations demonstrate the role of $\mathrm{J}$ in the very strong susceptibility of EuS to external fields or fields originating from direct exchange coupling to magnetic layers with a high $\mathrm{T}_{\mathrm{C}}$.

\section{Experimental Details}

One $\mathrm{Co} / \mathrm{EuS}$ and one Fe/EuS multilayers were deposited on the native oxide of $\mathrm{Si}(100)$ wafers in an ultrahigh vacuum (UHV) chamber by BALZERS. The base pressure was $4 \times 10^{-9}$ mbar. The deposition temperature was about $100{ }^{\circ} \mathrm{C}$. The growth was started and ended with EuS. No additional capping layer was used as EuS is stable in air. The deposition method for the EuS was e-beam sublimation: EuS powder of high purity was placed inside a tungsten crucible and the latter one in a water-cooled e-gun body. A weak e-beam of $25 \mathrm{~mA}$ had to be scanned on the surface of the powder in order to get a deposition rate of $0.05 \mathrm{~nm} / \mathrm{sec}$. The material sublimed directly from the target. Co and Fe were deposited by regular e-beam evaporation with a rate of about $0.03 \mathrm{~nm} / \mathrm{sec}$. The EuS, Co and Fe individual layer thickness was kept at about $4 \mathrm{~nm}$ in order comparison of the magnetic properties of the two multilayers to be meaningful. The number of periods $\mathrm{N}=6$ for both multilayers.

The layering quality and bilayer thickness were evaluated with the aid of the X-ray Reflectivity (XRR) technique. The XRR measurements were carried out using a Bruker 
Discover D8 reflectometer, equipped with a Gobel mirror, using $\mathrm{CuK} \alpha$ radiation $(\lambda=1.5418$ $\AA$ ). Structural details were revealed with the help of a JEOL 2011 High-Resolution Transmission Electron Microscope (HRTEM) operating at $200 \mathrm{kV}$, with a point resolution of $0.23 \mathrm{~nm}$ and $C s=1.0 \mathrm{~mm}$. Cross-section electron microscopy (XTEM) specimens were prepared by mechanical polishing, followed by ion milling in mild conditions.

Superconducting quantum interference device (SQUID) magnetometry measurements of the multilayers were performed at temperatures $\mathrm{T}=5-300 \mathrm{~K}$ under a maximum field of 3 T applied on the film plane. An MPMS XL - 7 SQUID magnetometer was used for this purpose. The measurements included recording of hysteresis loops at $5 \mathrm{~K}$ and $300 \mathrm{~K}$ and magnetization curves $\mathrm{M}(\mathrm{T})$ as a function of temperature. The contribution of the diamagnetic background, coming from the $\mathrm{Si}(001)$ wafer when one performs in-plane measurements was measured and removed.

\section{Results and Discussion}

In Fig. 1 we plot the XRR patterns for the two multilayers as indicated. The patterns consist of Bragg peaks, which satisfy the Bragg condition for the multilayer supercell. The first Bragg peak is pronounced for both multilayers. The second and third peaks fade away. The position of the respective Bragg peaks is practically the same, as one would have expected for multilayers of the same period length $\Lambda$. Between the Bragg peaks many Kiessig fringes appear. ${ }^{19}$ Analyzing the positions of successive Kiessig fringes the total film thickness $t$ may be accurately determined without the knowledge of the total reflection angle. The equation for the determination of the thin film thickness $t$ is then:

$$
2 t\left(\sin \theta_{n+1}-\sin \theta_{n}\right)=\lambda \quad,
$$

where $\theta_{n+1}$ and $\theta_{n}$ are the positions of two successive Kiessig fringes. The thickness $t$ of the multilayers are about 52-55 $\mathrm{nm}$. 
Figure 1 shows that the Bragg peaks after the first fade away and the Kiessig fringes appear only in small angles and then disappear. Both these indicate not perfect growth and the presence of roughness. XRR reveals also that $\mathrm{Co} / \mathrm{EuS}$ is better formed than $\mathrm{Fe} / \mathrm{EuS}$. Figure 2 confirms this result. Both samples have a peak-to-peak surface roughness of about 4-5 nm. The individual layers of $3 d$ metal and EuS are slightly thinner in the Fe/EuS multilayer than in $\mathrm{Co} / \mathrm{EuS}$. The layers in both samples show a patchy morphology. Co/EuS has better layer quality than $\mathrm{Fe} / \mathrm{EuS}$ in agreement with the XRR result. The Co or Fe layers comprise of nanocrystallites with size equal to the layer thickness. In addition, the EuS layers, interspaced between the $\mathrm{Co}$ or $\mathrm{Fe}$ ones, are up to $4.1 \mathrm{~nm}$ thick and exhibit a patchy morphology, too, consisting of small EuS grains.

To investigate the crystallographic structure, selected area diffraction patterns (SAD) were recorded for both samples, as shown in Fig. 3. X-ray diffraction (XRD) patterns, on the other hand, were inconclusive because they were noisy due to the small total film thickness. The SAD pattern on the top was obtained by an area of the whole Co/EuS multilayer and the Si substrate, the latter being in the [110] orientation. The ring pattern superimposed with the Si reflections denotes the multicrystalline nature of the Co and EuS layers. Furthermore, reflections attributed to the hexagonal Co and cubic EuS phases are also illustrated. The SAD pattern on the bottom stems from a region of the Fe/EuS multilayer and the Si substrate, too. Extra reflections in the pattern on the bottom are attributed to the Fe individual grains, as well as the EuS ones.

In Fig. 4 a HRTEM image of the Co/EuS multilayer is presented. The EuS grains often exhibited $\{111\}$-type lattice fringes, with a spacing of $\mathrm{d}=0.342 \mathrm{~nm}$. Furthermore, only a small percentage of crystallites were found whose lattice fringe separation was $d=0.347 \mathrm{~nm}$ and can be attributed to a Eu3 $\mathrm{S}_{4}$ phase. In rare occasions, some crystallites were also observed, such as the ones denoted with the white arrow in Fig. 4, where the lattice fringes have an average 
spacing of $\mathrm{d}=0.297 \mathrm{~nm}$ and can be assigned to the (200) planes of EuS. Similar results for $\mathrm{Fe} / \mathrm{EuS}$ showed that the EuS grains often exhibit $\{111\}$ - or $\{200\}$-type lattice fringes, having a spacing of $\mathrm{d}_{111}=0.342 \mathrm{~nm}$ or $\mathrm{d}_{200}=0.299 \mathrm{~nm}$, respectively. In rare occasions, crystallites were detected inside the EuS layers with a slightly higher spacing of $\mathrm{d}=0.348 \mathrm{~nm}$, which can be assigned to the one of the $\{211\}$ crystal planes of Eu3 $\mathrm{S}_{4}$ phase.

Figure 5 shows the room temperature hysteresis loops for the two multilayers. The shape of the magnetization curves is similar. Co and Fe show decreased magnetization by about $15 \%$ as compared to the bulk. ${ }^{20}$ The uncertainty in the volume determination of Co or $\mathrm{Fe}$ is at least $10 \%$. Moreover, one can not exclude a negative contribution from a small EuS magnetic moment oriented antiparallel to the $\mathrm{Co}$ or $\mathrm{Fe}$ one. Magnetic measurements with element specificity are needed to investigate this subject. The remanent magnetization is decreased as compared to the saturation magnetization in both samples. This could be due to some roughness and non complete continuity of $3 d$ metallic layers or due to EuS magnetic contribution. The fact that the $\mathrm{Fe} / \mathrm{EuS}$ with the worse layering has presents lower remanence than the $\mathrm{Co} / \mathrm{EuS}$ one witnesses for a potential structural origin of the decreased remanence.

Figure 6 shows the magnetization hysteresis loops of (a) Co/EuS and (b) $\mathrm{Fe} / \mathrm{EuS}$ multilayers recorded at $5 \mathrm{~K}$ (open symbols with dotted lines). The loops were recorded with a maximum field of $30 \mathrm{kOe}$ to ensure magnetic saturation, but they are presented up to $10 \mathrm{kOe}$ for better clarity. Since both, $3 d$ and EuS layers, are magnetic, the magnetization is normalized to the total film volume. $4 \mathrm{~nm}$ of $\mathrm{Co}$ or Fe can be considered as bulk-like with $\mathrm{T}_{\mathrm{C}}$ much higher than the room temperature. In this case it is reasonable to assume that the Co and Fe magnetizations have a very weak temperature dependence between 300 and $5 \mathrm{~K}^{20}$ Then one may subtract from the total magnetic signal the $3 d$ contribution and estimate the EuS magnetization at $5 \mathrm{~K}$. The result is only about $(650 \pm 15 \%) \mathrm{emu} / \mathrm{cm}^{3}$. This value corresponds to about $3.8 \mu_{\mathrm{B}} /$ atom to be compared with the literature value of about $6.9 \mu_{\mathrm{B}} /$ atom for bulk 
EuS. $^{21,22}$ It is interesting to notice here that it is not easy to compare our data with literature, because frequently either relative or not-normalized-per-volume values are provided for EuS/3 $d$ thin films. ${ }^{12,16,18}$ For EuS/PbS epitaxial superlattices the magnetic moment of EuS was measured to be equal to the bulk value. ${ }^{23}$ On the other extreme, for EuS nanoparticles a strongly decreased value of magnetization was reported. ${ }^{24}$ Element-specific measurements of Ref. [24] and recent ones of ours on Co/EuS multilayers, ${ }^{25}$ raise the problem of valency. This is described in detail by x-ray photoemission spectroscopy in Ref. [13]. Namely, it was shown that in EuS layers in layered systems there was coexistence of $\mathrm{Eu}^{++}$and $\mathrm{Eu}^{+++}$, with the latter one having $\mathrm{J}=0$ and carrying no magnetic moment. The temperature of growth was found to be an important parameter. $400{ }^{\circ} \mathrm{C}$ was found to be a good temperature for the elimination of the problem. Between R.T. and $400{ }^{\circ} \mathrm{C}$ the ratio of divalent-to-trivalent Eu was increasing. ${ }^{13}$ However, practically temperatures above $200{ }^{\circ} \mathrm{C}$ usually degrade the multilayer quality. Therefore, it seems that one has always to face the valency problem. Our electron microscopy work verifies the Smits arguments that the valency problem is mainly a problem of defects and not of extended $\mathrm{Eu}_{3} \mathrm{~S}_{4}$ phase formation. The decreased average magnetic moment of 3.8 $\mu_{\mathrm{B}} /$ atom found in this work declares that the ratio of divalent-to-trivalent $\mathrm{Eu}$ is slightly larger than one and is in fair agreement with Ref. [13].

In Fig. 6 the thick lines are calculated loops for the magnetization of $\mathrm{Co} / \mathrm{EuS}$ and Fe/EuS multilayers based on a Stoner-Wohlfarth-like model. ${ }^{14,15}$ In our calculations we used the magnetization values of $3 d$ and EuS of our measurements and allowed them to vary within $10 \%$. Besides the use of a direct bilinear antiferromagnetic exchange coupling, which is known to hold between $3 d$ and $4 f$ magnetic atoms, ${ }^{26,27}$ we used a biquadratic coupling in order to have best fit of the experimental data. As origin of it could be the non-collinearities of the magnetic moments at the interfaces due to roughness, similar, for example, to the cases of directly coupled exchange biased systems or interlayer exchange coupling. ${ }^{28,29}$ The free energy 
density of the system is then given by: ${ }^{16,30,31}$

$$
\mathrm{F}=-\mathrm{J}_{1} \cos \Delta \theta-\mathrm{J}_{2} \cos (2 \Delta \theta),
$$

with $\Delta \theta$ being the angle between the magnetizations of $3 d$ and EuS layers on the film plane. $\mathrm{J}_{1}$ and $\mathrm{J}_{2}$ are the bilinear and biquadratic exchange coupling constants. After normalization to the thickness of the magnetic layers in order to be able to compare to literature values, ${ }^{30,32}$ we find from our fitting: $\mathrm{J}_{\mathrm{AF}}=0.38 \mathrm{erg} / \mathrm{cm}^{3}$ for $\mathrm{Co} / \mathrm{EuS}$ and $\mathrm{J}_{\mathrm{AF}}=0.45 \mathrm{erg} / \mathrm{cm}^{3}$ for $\mathrm{Fe} / \mathrm{EuS}$. These values are significantly larger than ones reported previously for $\mathrm{Co} / \mathrm{EuS}$ and $\mathrm{Fe} / \mathrm{EuS}$ bilayers. ${ }^{16-18}$ This can be directly seen by our much larger saturation fields than the ones of previous reports. ${ }^{16-18}$ The strength of the biquadratic coupling was almost one order of magnitude smaller than that of the direct bilinear antiferromagnetic exchange coupling.

In Fig. 7 we plot the temperature-dependend magnetizations of our samples under an external field of $20 \mathrm{kOe}$ applied parallel to the film plane. One may observe a strong magnetic response of EuS up to a temperature three times larger than the $\mathrm{T}_{\mathrm{C}}$ of bulk EuS. This is due to proximity effects to the $3 d$ layers. ${ }^{9-12,16-18}$ A tail of the magnetization of EuS seems to persist up to at least about $100 \mathrm{~K}$. One can not exclude the fact that some number of EuS layers at interfaces might be magnetic up to room temperature. For example, in the case of $\mathrm{CoF}_{2} / \mathrm{FeF}_{2}$ antiferromagnetic superlattices, three atomic layers of $\mathrm{CoF}_{2}$ at each interface maintain their spin polarization due to proximity to $\mathrm{FeF}_{2}$ at temperatures much higher than their critical temperature and up to about $80 \%$ of the critical temperature of $\mathrm{FeF}_{2} \cdot{ }^{33}$ In the same Figure we have calculated a potential paramagnetic response of EuS layers based on Brillouin functions. $^{20}$ The best fitting was found for $30 \mathrm{kOe}$ applied field. It is reasonable to think that over the $20 \mathrm{kOe}$ external field an effective exchange field of $10 \mathrm{kOe}$ is superimposed. As was shown earlier, both ferromagnetic and antiferromagnetic exchange coupling increase the critical temperature of magnetic layers. ${ }^{34,35}$

At first sight, the strong increase of ordering temperature of three dimensional 
ferromagnets by relatively small effective fields seems puzzling. ${ }^{36}$ In Fig. 8 we show similar calculations to the one of Fig. 7 for a magnetic system with $J=1 / 2$, e.g. Ni, and one with $J=$ $7 / 2(\mathrm{EuS})$. It is clearly demonstrated the role of the large $\mathrm{J}$ to the largest magnetic response of the system for temperatures higher than the ordering temperature. The spin polarization of EuS at R.T. for a field of $5 \mathrm{~T}(50 \mathrm{kOe})$ is about $4 \%$. This is in good agreement with experimental values on EuS nanoparticles and recent calculations for divalent europium nitride. $^{24,37}$

\section{Summary and perspectives}

In conclusion, we have investigated the growth and the temperature-dependent magnetic properties of two identical $\mathrm{Co} / \mathrm{EuS}$ and $\mathrm{Fe} / \mathrm{EuS}$ multilayers. The main results are: (i) Both multilayers have a relatively good growth, but with significant roughness. Co is hexagonal, while Fe and EuS are cubic. There is some $\{100\}$ texture of EuS crystallites. Crystallite size is limited by the individual layer thickness of $4 \mathrm{~nm}$. A very small amount of $\mathrm{Eu}_{3} \mathrm{~S}_{4}$ phase is formed. (ii) We observed a strong decrease of the EuS magnetization at $5 \mathrm{~K}$ due to the mixedvalent state of $\mathrm{Eu}$. Only $\mathrm{Eu}^{++}$contributes to ferromagnetism. (iii) We have determined via fitting of the magnetization curves at $5 \mathrm{~K}$ antiferromagnetic exchange coupling constant values much larger than previously reported. ${ }^{16-18}$ (iv) EuS layers are very susceptible to effective fields due to the $J=7 / 2$ character of the system. (v) Ferromagnetic response at R.T. can not be concluded from these results. If this occurs, then this, in combination with recently reported quantum confinement effects in the optical properties in very thin EuS layers, would render the system valuable for new optoelectronics and spintronics applications. ${ }^{38}$ (vi) The $\mathrm{J}=7 / 2$ character of the system is very promising for large $T_{C}$ enhancement under moderate exchange fields. The last step for strong ferromagnetic response at room temperature is to decrease the EuS layers to the two-dimensional limit. According to Jensen et al. spin 
fluctuations act as an amplifier of the exchange coupling effects on the enhancement of $\mathrm{T}_{\mathrm{C}}$ of the low $\mathrm{T}_{\mathrm{C}}$ magnetic layer when the latter approaches the two-dimensional limit. ${ }^{39,35}$ Indeed, very recent element-specific measurements demonstrate that $2 \mathrm{~nm}$ thick (approximately 6 atomic layers) EuS shows strong ferromagnetic response at room temperature due to proximity with $\mathrm{Co}$ in $\mathrm{Co} / \mathrm{EuS}$ multilayers, regenerating this way the interest for research on EuS based spintronics. ${ }^{25}$

Acknowledgements: This work has been partly supported by the Research Committee of the University of Patras, grant Karatheodori, Nr. C.905/2009. One of us (P.P.) thanks the Center for International Cooperation (C.I.C.) of the Freie Universität Berlin for partial financial support and the Institute for Experimental Physics, Freie Universität Berlin for the great hospitality. 


\section{References}

1. S. A. Wolf, D. D. Awschalom, R. A. Buhrman, J. M. Daughton, S. von Molnár, M. L. Roukes, A. Y. Chtchelkanova, and D. M. Treger, Science 294, 1488 (2001).

2. I. Žutić, J. Fabian and S. Das Sarma, Rev. Mod. Phys. 76, 323 (2004).

3. C. Felser, G.H. Fecher, and B. Balke, Angew. Chemie 46, 668 (2007).

4. G. Maruccio, J. Spintron. Magn. Nanomater. 1, 3 (2012).

5. Parmanand Sharma, Amita Gupta, K. V. Rao, Frank J. Owens, Renu Sharma, Rajeev Ahuja, J. M. Osorio Guillen, Börje Johansson and G. A. Gehring, Nature Materials 2, $673(2003)$.

6. A. Ney, R. Rajaram, E. Arenholz, J. S. Harris, Jr., M. Samant, R. F. C. Farrow and S.S.P. Parkin, J. Magn. Magn. Mat. 300, 7 (2006).

7. A. Ney, K. Ollefs, S. Ye, T. Kammermeier, V. Ney, T.C. Kaspar, S.A. Chambers, F. Wilhelm and A. Rogalev, Phys. Rev. Lett. 100, 157201 (2008).

8. V. Ney, S. Ye, K. Ollefs, T. Kammermeier, F. Wilhelm, A. Rogalev and A. Ney, J. Nanosci. Nanotechnol. 10, 5958 (2010).

9. P. Fumagalli, A. Schirmeisen and R.J. Gambino, Phys. Rev. B 57, 14294 (1998).

10. C. Müller, H. Lippitz, J.J. Paggel and P. Fumagalli, J. Appl. Phys. 95, 7172 (2004).

11. C. Müller, H. Lippitz, J.J. Paggel and P. Fumagalli, J. Appl. Phys. 99, 073904 (2006).

12. V.V. Volobuev, A.N. Stetsenko and J. van Lierop, J. Appl. Phys. 103, 07C905 (2008).

13. C.J.P. Smits, EuS based thin film layered systems, PhD Thesis, TU-Delft (2006) unpublished.

14. P. Poulopoulos, R. Krishnan and N.K. Flevaris, J. Magn. Magn. Mat. 163, 27 (1996).

15. E. Th. Papaioannou, M. Angelakeris, N.K. Flevaris, P. Fumagalli, Ch. Müller, A. Troupis, A. Spanou, V. Karoutsos, P. Poulopoulos, V. Kapaklis and C. Politis, J. Appl. Phys. 101, 023913 (2007). 
16. U. Rücker, S. Demokritov, R.R. Arons and P. Grünberg, J. Magn. Magn. Mat. 156, 269 (1996).

17. M. Szot, L. Kowalczyk, T. Story, V. Domukhovski, W. Knoff, K. Gas, V.V. Volobuev, A. Yu. Sipatov, and A. G. Fedorov, Acta Phys. Pol. A 114, 1397 (2008).

18. V.V. Volobuev, A.N. Stetsenko, A. Yu Sipatov and J. van Lierop, Phys. Rev. B 81, $134430(2010)$

19. H. Kiessig, Ann. Phys. (Leipzig) 10, 715 (1931).

20. Ch. Kittel, Introduction to Solid State Physics, $5^{\text {th }}$ edition, Wiley \& Sons, New York (1976).

21. A. Mauger and C. Godart, Physics Reports 141, 51 (1986).

22. T. Jeong, Intern. J. Mod. Phys. B 26, 1250150 (2012).

23. C.J.P. Smits, A.T. Filip, H.J.M. Swagten, W.J.M. de Jonge, M. Chernyshova, L. Kowalczyk, K. Grasza, A. Szczerbakow, T. Story and A. Yu. Sipatov, J. Appl. Phys. 95, 7169 (2004).

24. Rachel S. Selinsky, David J. Keavney, Matthew J. Bierman and Song Jin, Appl. Phys. Lett. 95, 202501 (2009).

25. S.D. Pappas, P. Poulopoulos, B. Lewitz, A. Straub, A. Goschew, V. Kapaklis, F. Wilhelm, A. Rogalev and P. Fumagalli (submitted).

26. B. Scholz, R.A. Brand and W. Keune, Hyperf. Inter. 68, 409 (1992).

27. M.S.S. Brooks, L. Nordström and B. Johansson, J. Appl. Phys. 69, 5683 (1991).

28. J.C. Slonczewski, Phys. Rev. Lett. 67, 3172 (1991).

29. R.L. Stamps, J. Phys. D: Appl. Phys. 33, R247 (2000).

30. P. Poulopoulos, P. Isberg, W. Platow, W. Wisny, M. Farle, B. Hjörvarsson and K. Baberschke, J. Magn. Magn. Mater. 170,57 (1997).

31. D.E. Bürgler, P. Grünberg, S.O. Demokritov and M.T. Johnson, in Hanbook of 
Magnetic Materials, K.H.J. Buschowe (ed.), Vol.13, pp. 1-85, Elsevier Science B.V. (2001).

32. $\quad$ S.S.P. Parkin, Phys. Rev. Lett. 67, 3598 (1991).

33. A.S. Carriço and R.E. Camley, Phys. Rev. B 45, R13117-13120 (1992).

34. G. Bayreuther, F. Bensch and V. Kottler, J. Appl. Phys. 79, 4509 (1996).

35. U. Bovensiepen, F. Wilhelm, P. Srivastava, P. Poulopoulos, M. Farle, A. Ney and K. Baberschke, Phys. Rev. Lett. 81, 2368 (1998).

36. P. Poulopoulos, M. Farle, U. Bovensiepen and K. Baberschke, Phys. Rev. B 55, R11961 (1997).

37. B. J. Ruck, H. J. Trodahl, J. H. Richter, J. C. Cezar, F.Wilhelm, A. Rogalev, V. N. Antonov, Binh Do Le and C. Meyer, Phys. Rev. B 83, 174404 (2011).

38. P. Poulopoulos, B. Lewitz, A. Straub, S.D. Pappas, S.A. Droulias, S. Baskoutas and P. Fumagalli, Appl. Phys. Lett. 100, 211910 (2012).

39. P.J. Jensen, K.H. Bennemann, P. Poulopoulos, M. Farle, F. Wilhelm and K. Baberschke, Phys. Rev. B 60, R14994 (1999). 


\section{Figure Captions}

\section{Figure 1}

X-ray reflectivity patterns for the $\mathrm{Co} / \mathrm{EuS}$ and the $\mathrm{Fe} / \mathrm{EuS}$ multilayers grown on the native amorphous oxide of a $\mathrm{Si}(100)$ wafer. The pattern of $\mathrm{Fe} / \mathrm{EuS}$ has been vertically shifted (multiplied by 0.001 ) for clarity of presentation.

\section{Figure 2}

(top) XTEM of the Co/EuS multilayer (bottom) XTEM of the Fe/EuS multilayer. The surface roughness is about $4-5 \mathrm{~nm}$.

Figure 3

(top) SAD pattern of the $\mathrm{Co} / \mathrm{EuS}$ multilayer (bottom) SAD of the Fe/EuS multilayer. Co crystallites are hexagonal while Fe ones are cubic. Diffractions from $\operatorname{EuS}(200)$ and $\operatorname{EuS}(111)$ are visible.

Figure 4

HRTEM from the Co/EuS multilayer. The EuS crystallites are limited in size by the EuS layer thickness. (111) and (100) EuS crystallites are observed. Some crystallites have crystal plane distances which fit to (211) $\mathrm{Eu}_{3} \mathrm{~S}_{4}$.

Figure 5

(a) SQUID magnetization curve for the Co/EuS multilayer at room temperature (b) SQUID magnetization curve for the $\mathrm{Fe} / \mathrm{EuS}$ multilayer at room temperature. 
Figure 6 (color on-line)

(a) SQUID magnetization curve for the $\mathrm{Co} / \mathrm{EuS}$ multilayer at $\mathrm{T}=5 \mathrm{~K}$ (b) SQUID magnetization curve for the $\mathrm{Fe} / \mathrm{EuS}$ multilayer at $\mathrm{T}=5 \mathrm{~K}$. The thick lines are the results of calculations for antiferromagnetically coupled $3 d$ and EuS layers.

Figure 7 (color on-line)

$\mathrm{M}(\mathrm{T})$ curves under an external in-plane field of $2 \mathrm{~T}$ for the $\mathrm{Co} / \mathrm{EuS}$ and $\mathrm{Fe} / \mathrm{EuS}$ multilayers as indicated. The solid lines are the results of calculations based on a Brillouin function magnetic response of the EuS layers above $\mathrm{T}_{\mathrm{C}}$ under a total field of $3 \mathrm{kOe}$.

Figure 8 (color on-line)

Calculated $\mathrm{M}(\mathrm{T})$ curves under various fields as indicated for magnetic layers of a system with $\mathrm{J}=7 / 2$ and a system with $\mathrm{J}=1 / 2$. The calculations are Brillouin function magnetic responses. The $\mathrm{J}=7 / 2$ is very susceptible to fields justifying the enhanced $\mathrm{T}_{\mathrm{C}}$ of $\mathrm{EuS}$ in proximity to ferromagnetic $3 \mathrm{~d}$ layers 


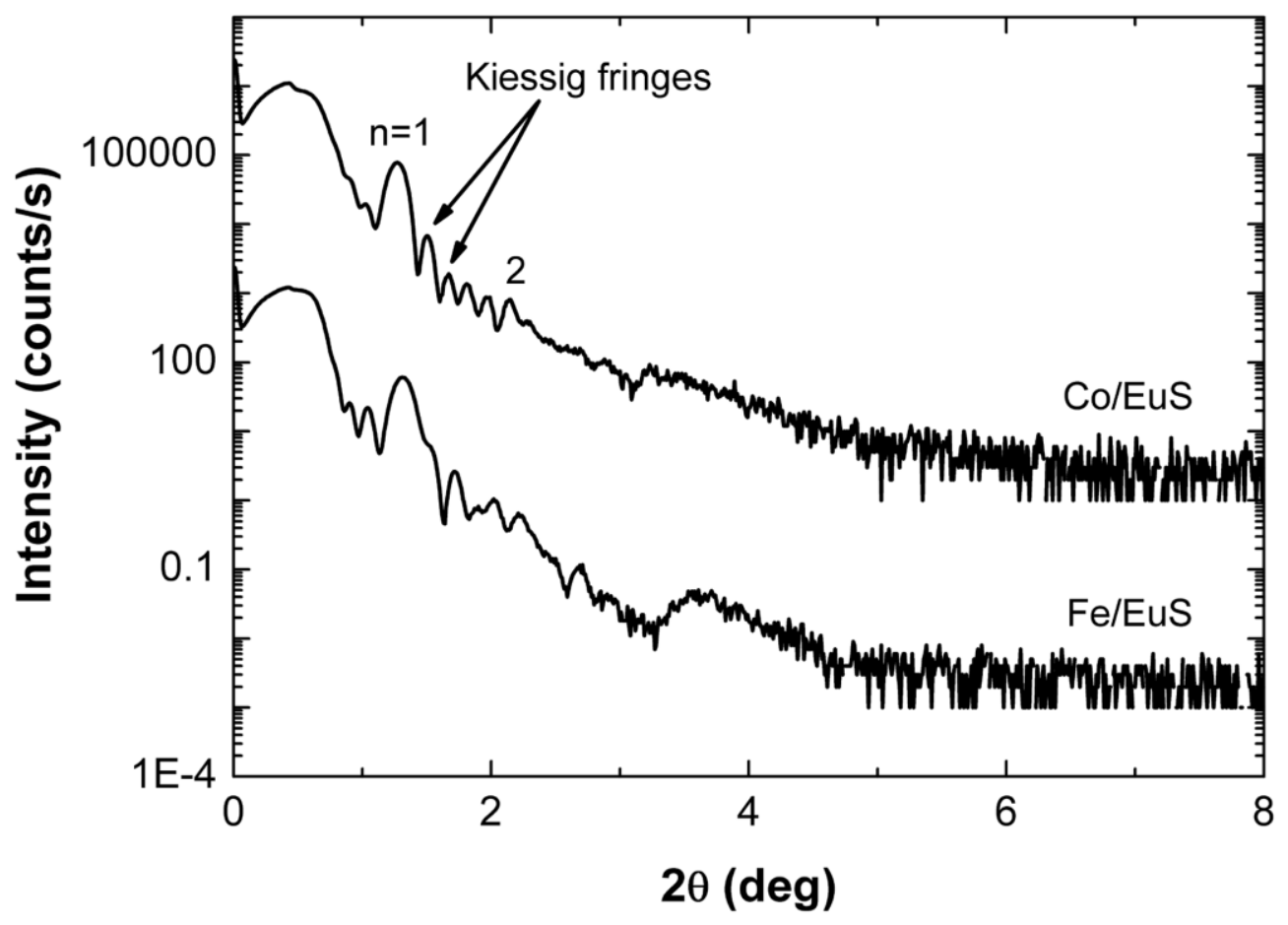

Figure 1, Lewitz et al.

PROXIMITY EFFECTS AND CURIE TEMPERATURE ENHANCEMENT IN Co/EUS AND Fe/EUS MULTLAYYERS 


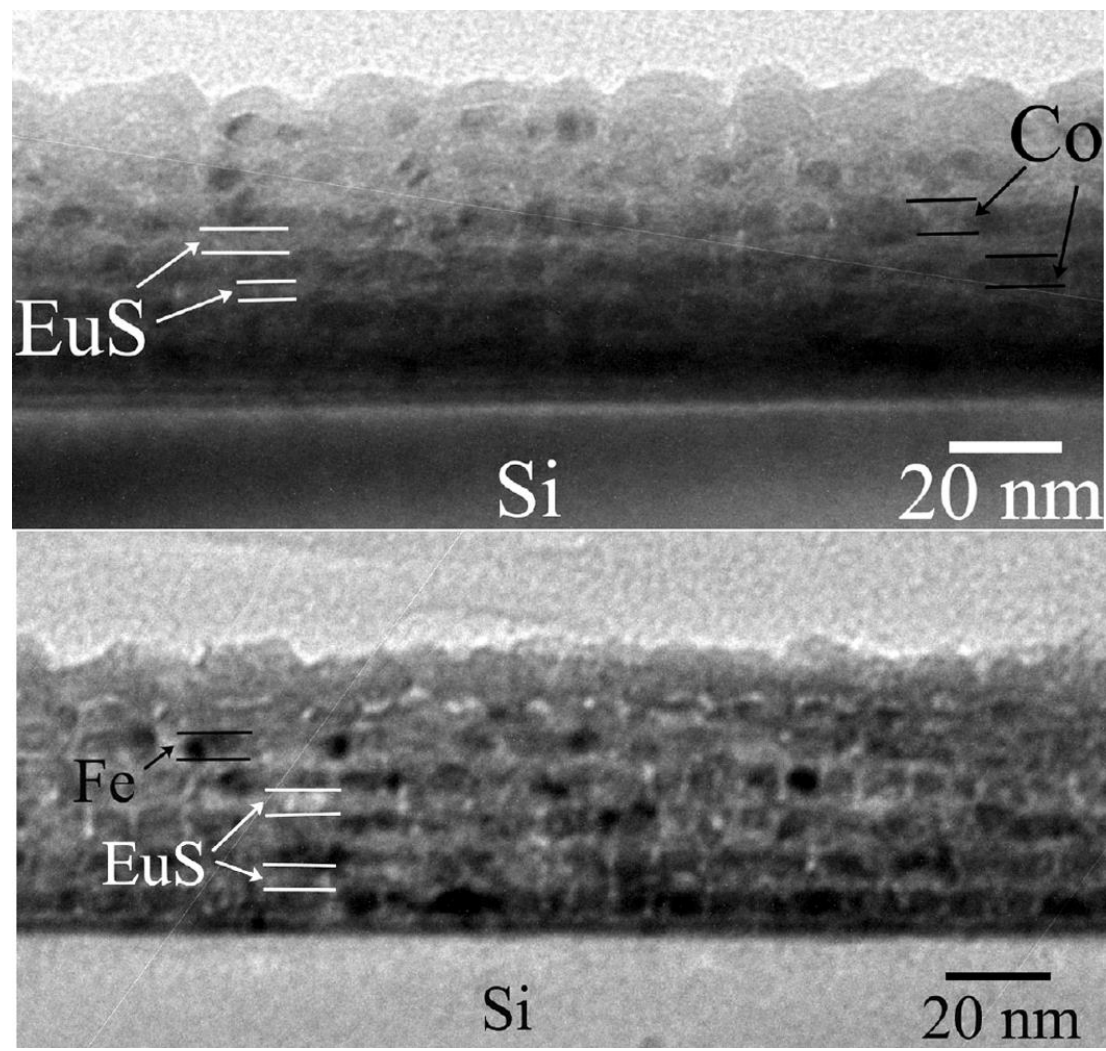

Figure 2, Lewitz et al.

PROXIMITY EFFECTS AND CURIE TEMPERATURE ENHANCEMENT IN Co/EUS AND Fe/EuS MULTILAYERS 


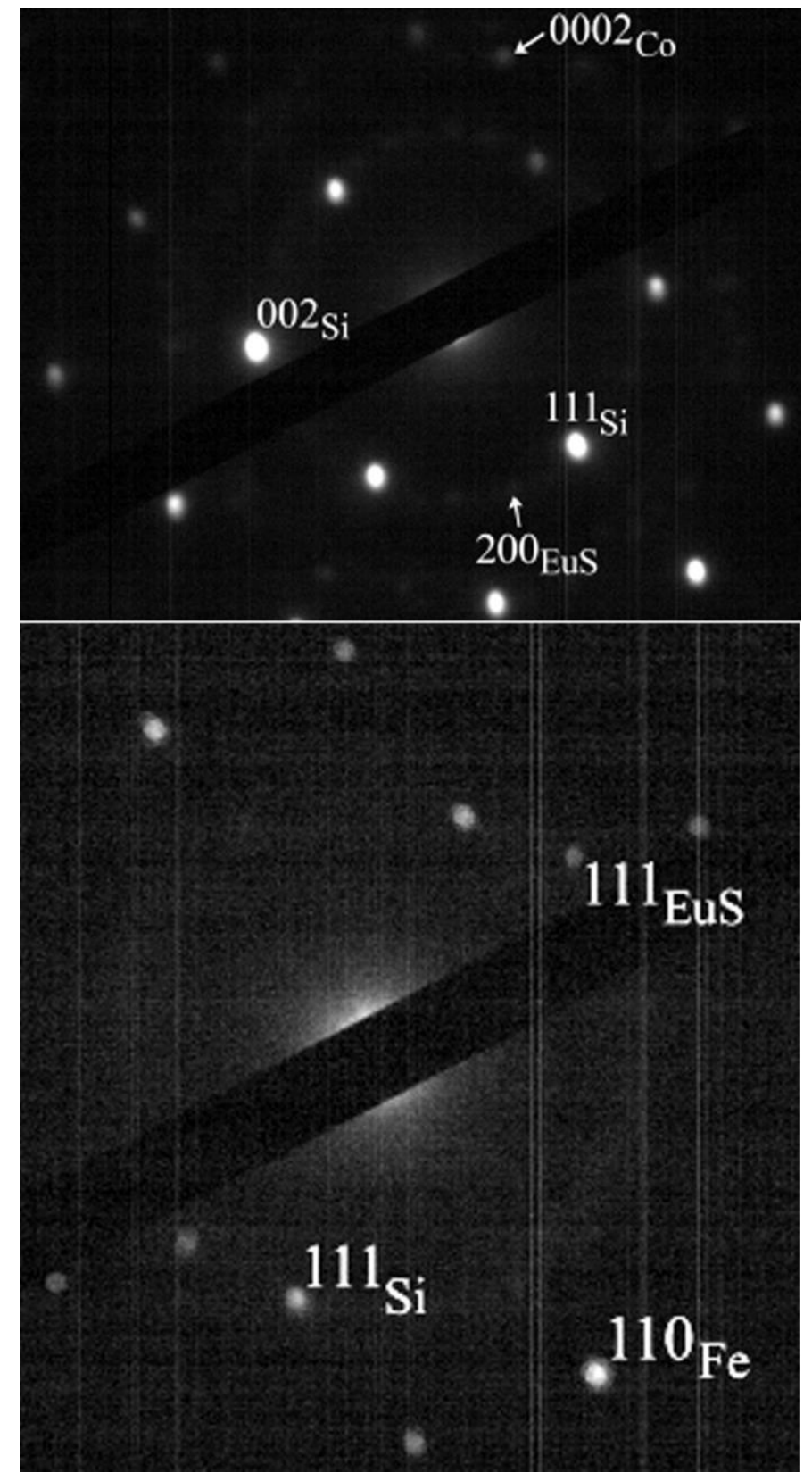

Figure 3, Lewitz et al.

PROXIMITY EFFECTS AND CURIE TEMPERATURE ENHANCEMENT IN Co/EuS AND Fe/EuS MULTILAYERS 


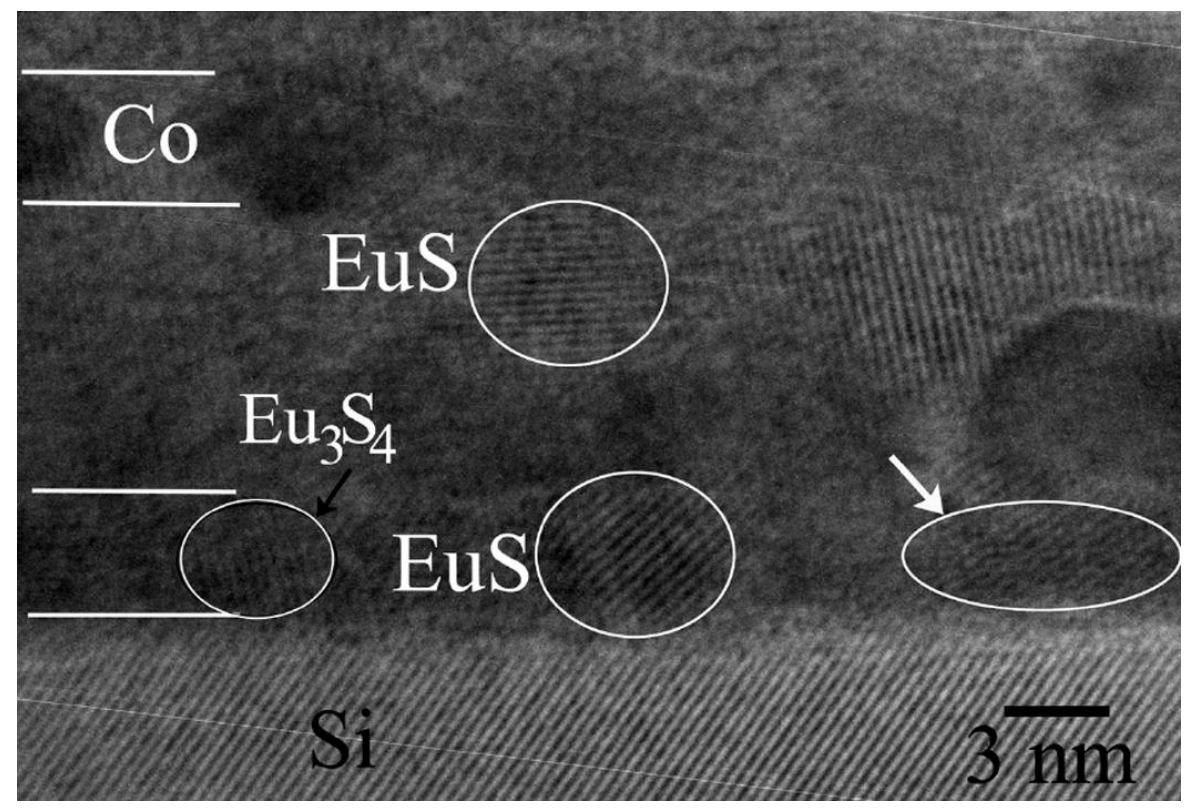

Figure 4, Lewitz et al.

PROXIMITY EFFECTS AND CURIE TEMPERATURE ENHANCEMENT IN Co/EUS AND Fe/EuS MULTILAYERS 

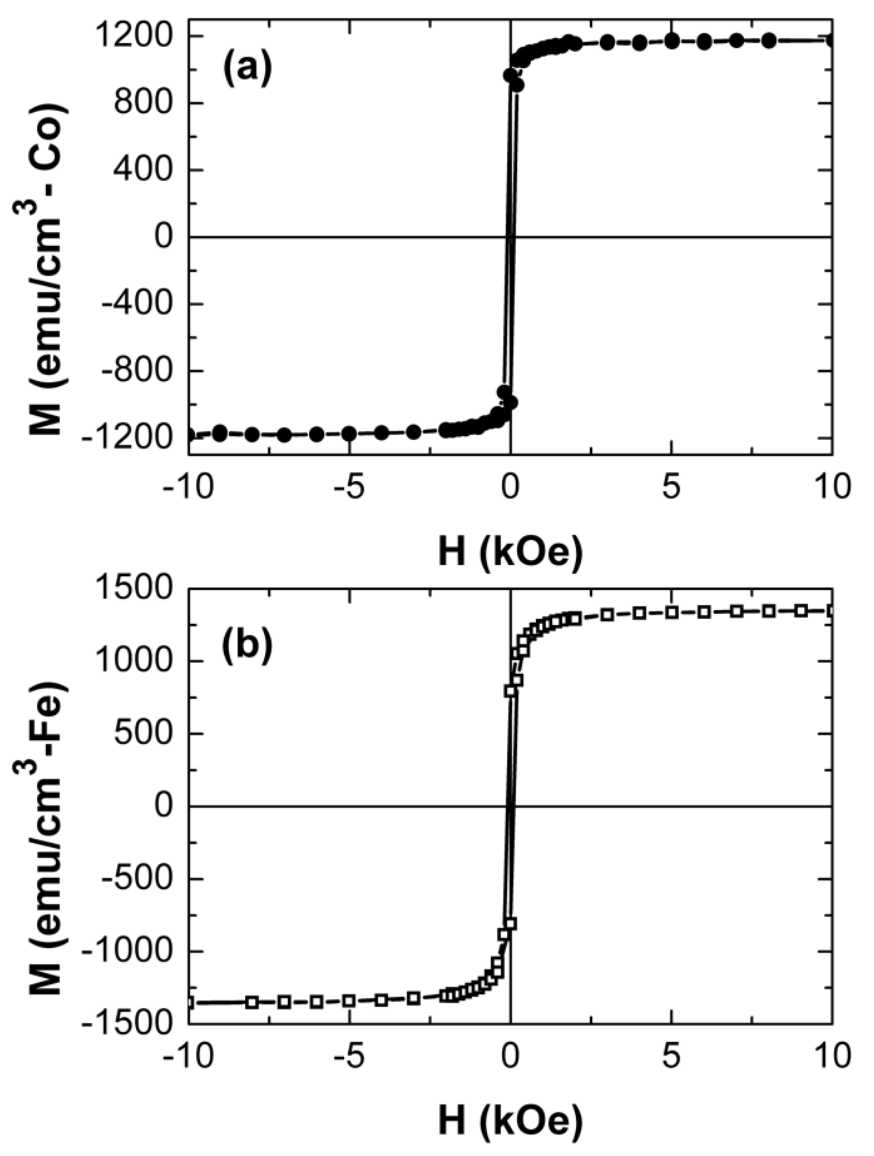

Figure 5, Lewitz et al.

PROXIMITY EFFECTS AND CURIE TEMPERATURE ENHANCEMENT IN Co/EUS AND Fe/EuS MULTILAYERS 

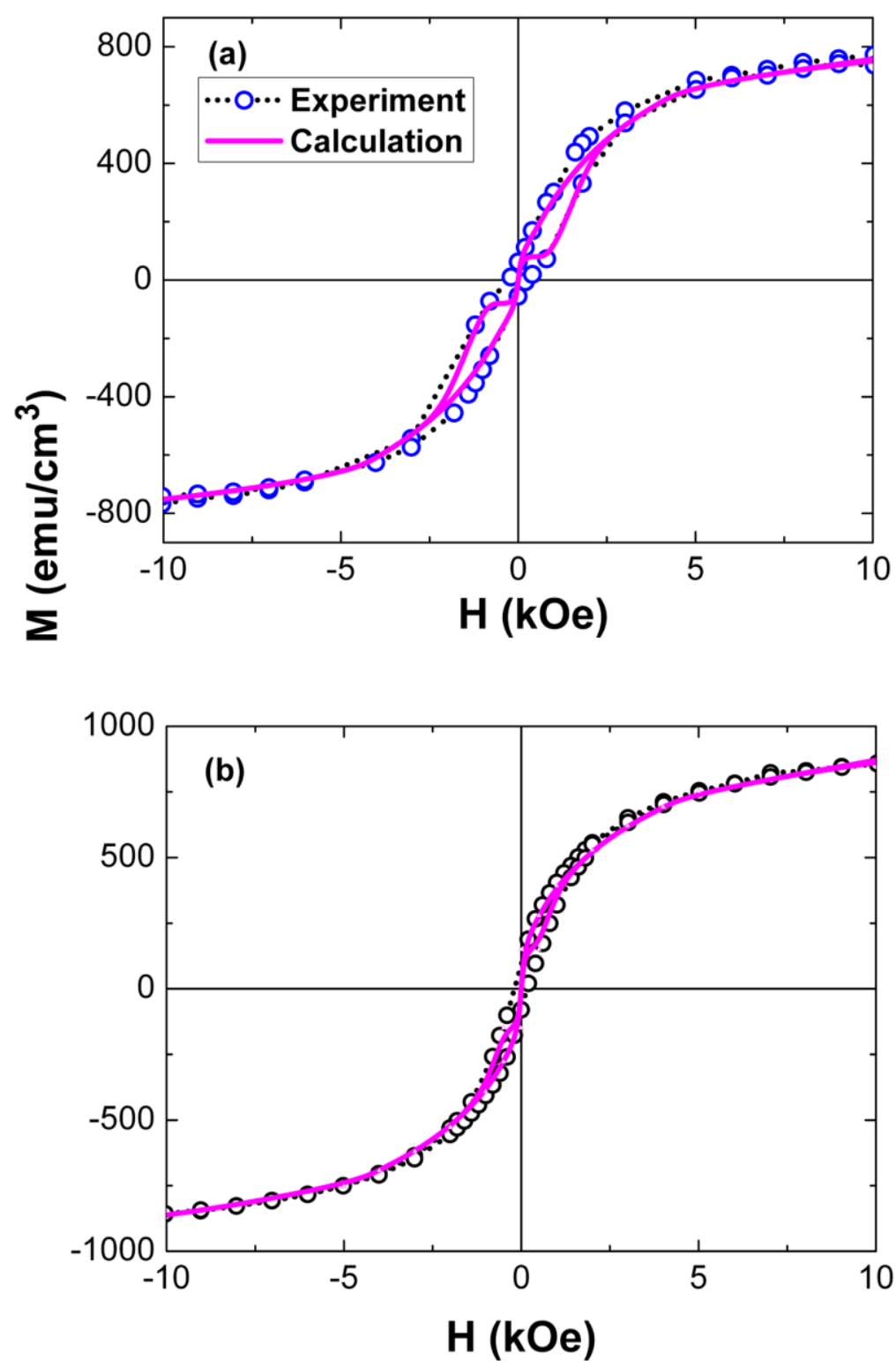

Figure 6, Lewitz et al.

PROXIMITY EFFECTS AND CURIE TEMPERATURE ENHANCEMENT IN Co/EUS AND Fe/EUS MULTILAYERS 


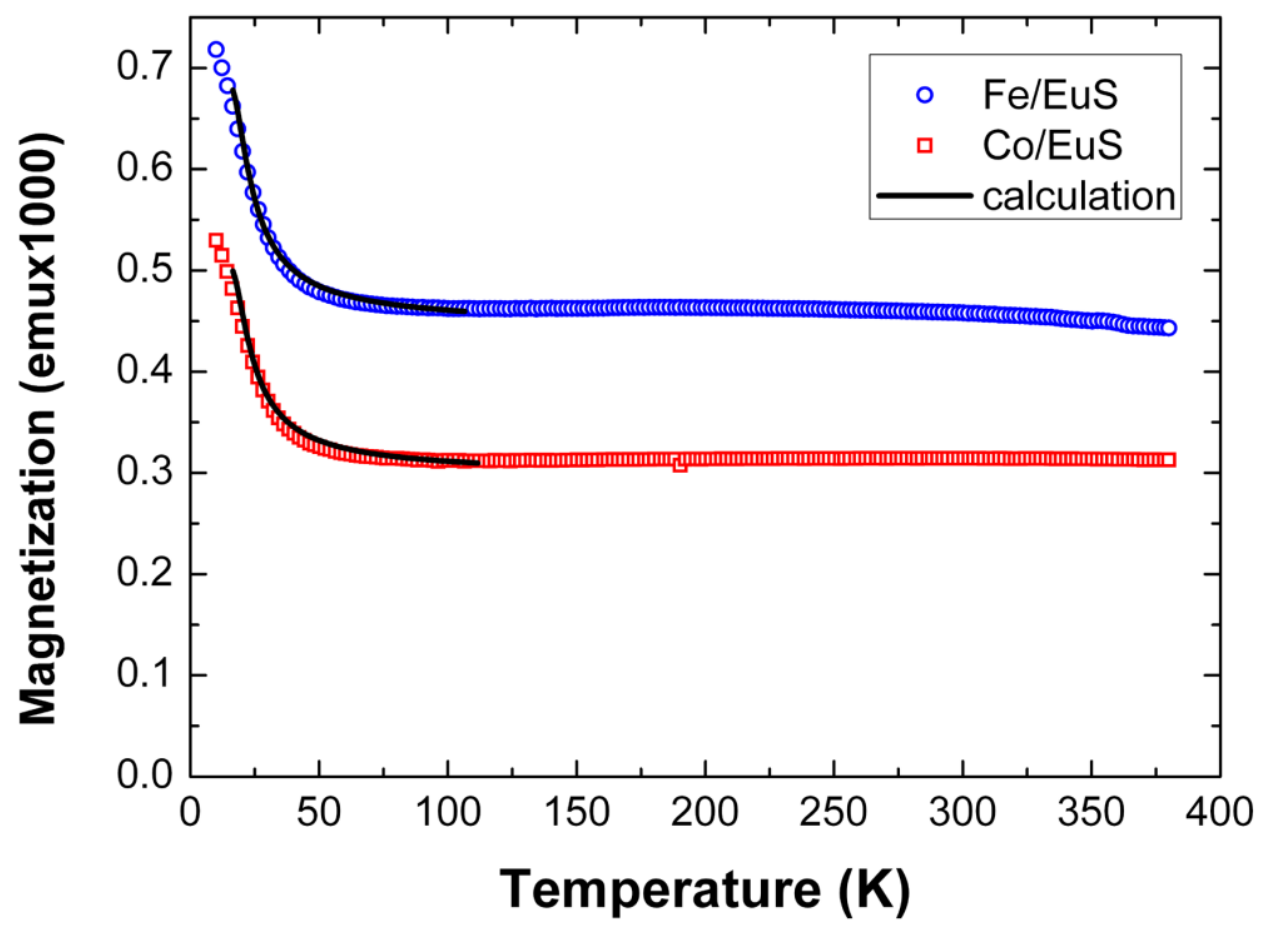

Figure 7, Lewitz et al.

PROXIMITY EFFECTS AND CURIE TEMPERATURE ENHANCEMENT IN Co/EUS AND Fe/EuS MULTILAYERS 


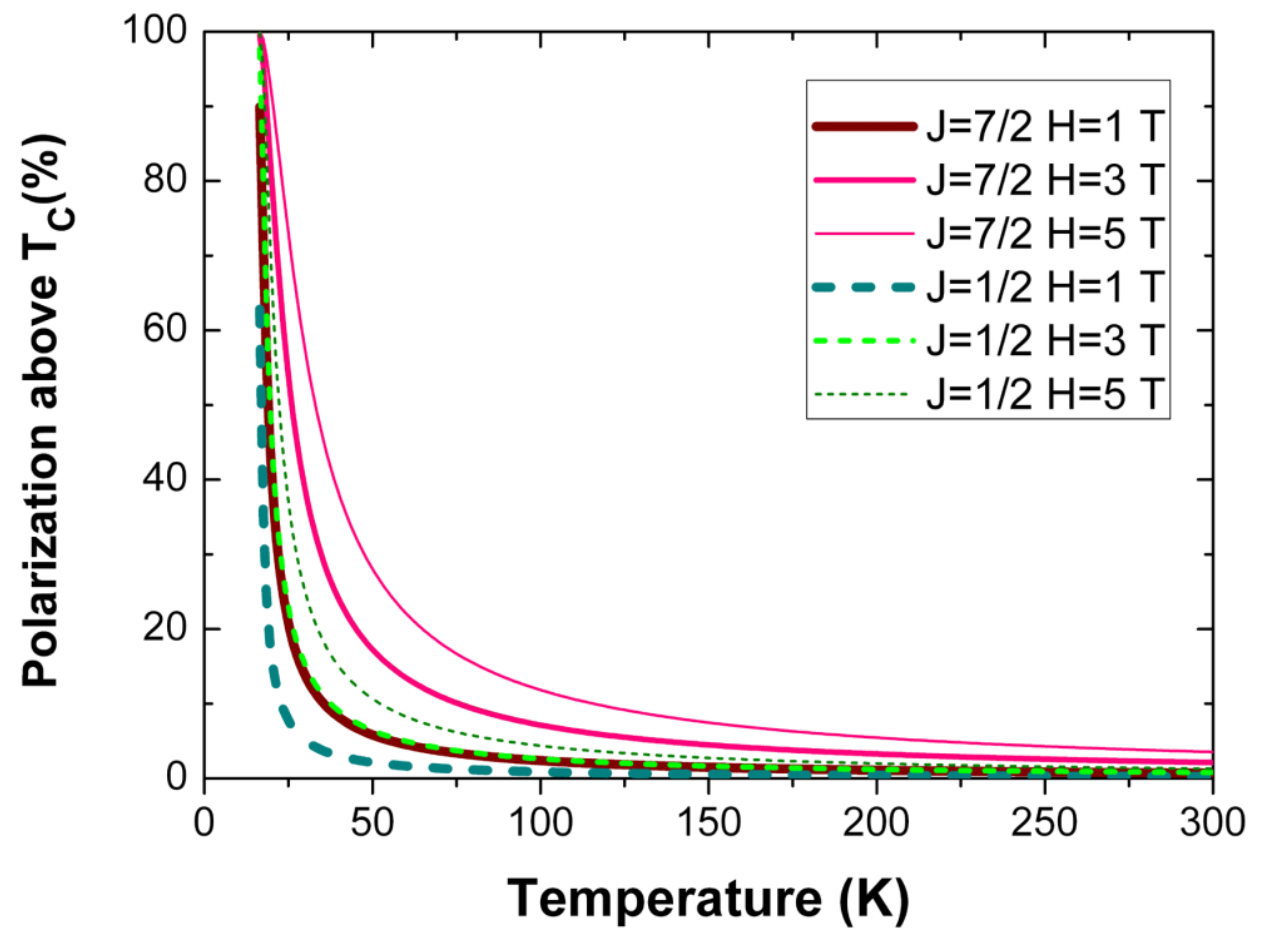

Figure 8, Lewitz et al.

PROXIMITY EFFECTS AND CURIE TEMPERATURE ENHANCEMENT IN Co/EUS AND Fe/EuS MULTILAYERS 